\title{
Carboplatin/Paclitaxel/Nivolumab Regimen
}

National Cancer Institute

\section{Source}

National Cancer Institute. Carboplatin/Paclitaxel/Nivolumab Regimen. NCI Thesaurus.

Code C156211.

A regimen consisting of carboplatin, paclitaxel and nivolumab that can potentially be used for the treatment of various cancers, such as head and neck squamous cell carcinoma and ovarian cancer. 